\title{
Gliomatosis cerebri
}

\section{Orale Chemotherapie auf dem Prüfstand}

Glas M, Bähr O, Felsberg $\mathrm{J}$ et al. NOA-05 Phase 2 Trial of Procarbazine and Lomustine therapy in Gliomatosis cerebri. Ann Neurol 2011; 70: $445-53$

Abbildung 1 Zeit bis zum Therapieversagen (FFS) bei 35 Patienten mit Gliomatosis cerebri bei Behandlung mit Procarbazin und CCNU.
Fragestellung: Es gibt keine Standardtherapie der Gliomatosis cerebri. Kann diese Erkrankung mit einer oralen Chemotherapie aufgehalten werden?

Hintergrund: Die Gliomatosis cerebri ist eine Gliomerkrankung des Gehirns, die mindestens drei Hirnlappen befällt. Histologisch können diese Tumoren astrozytären Gliomen des WHO-Grades II oder III, seltener oligodendroglialen Tumoren oder auch Glioblastomen entsprechen. Eine Heilung ist nicht möglich: Aufgrund der Ausdehnung ist eine komplette oder auch nur weitgehende Tumorresektion nicht durchführbar. Eine Strahlentherapie (mit mindestens 45 Gy) umfasst immer

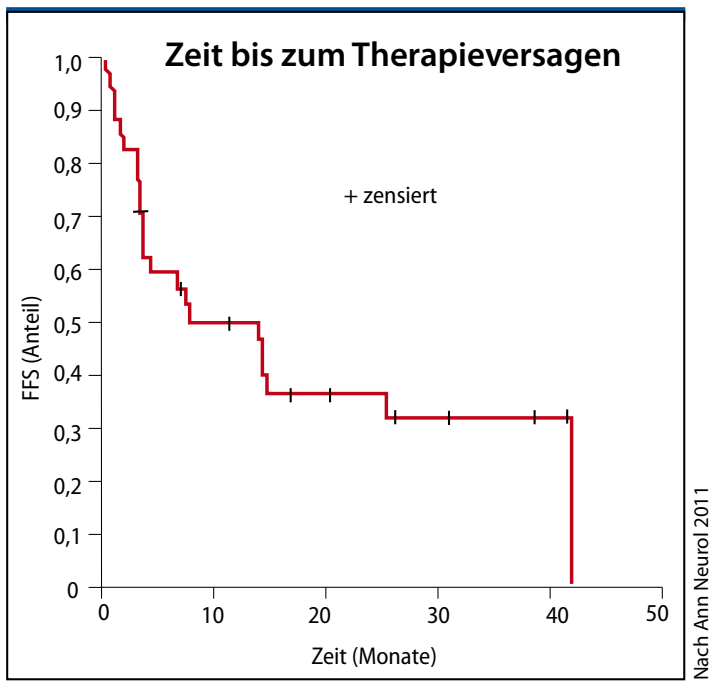

ein sehr großes Zielvolumen und wird wegen möglicher Neurotoxizität gefürchtet. Zur Chemotherapie gab es bislang nur Kasuistiken oder kleine prospektive Fallsammelserien.

Patienten und Methodik: Es handelte sich um eine einarmige Phase-II-Studie zur Chemotherapie mit Procarbazin und CCNU (PC) bei Patienten mit einer Gliomatosis cerebri, einem Lebensalter von über 18 Jahren und einem Karnofsky Performance Index (KPI) von mindestens 50. Das prädefinierte Ziel war es, einen Anteil von mindestens $30 \%$ der Patienten zu erreichen, die nach acht Monaten noch kein Therapieversagen aufwiesen (FFS, Failure-free survival).

CCNU 110 mg $/ \mathrm{m}^{2}$ Körperoberfläche (KOF) an Tag 1 und Procarbazin $60 \mathrm{mg} / \mathrm{m}^{2} \mathrm{KOF}$ pro Tag an den Tagen 8 bis 21 wurden oral alle acht Wochen für bis zu sechs Zyklen verabreicht.
Ergebnisse: Insgesamt wurden 35 Patienten in die Studie eingeschlossen: Einer starb so rasch, dass er keine Therapie mehr erhielt; bei zwei Patienten musste die Therapie wegen Toxizität abgesetzt werden, fünf weitere wiesen eine relevante (WHO-Grad III oder IV) Hämatotoxizität auf. Eine Größenabnahme des Tumors um mehr als $50 \%$ wurde bei fünf Patienten erreicht, kein Tumor zeigte eine komplette Remission. Die KaplanMeier-Analyse zeigte für den prädefinierten Endpunkt FFS bei acht Monaten einen kalkulierten Wert von $50,3 \%$, eine mediane FFS-Zeit von 14 Monaten (Abbildung 1) und eine mediane Gesamtüberlebenszeit von 30 Monaten. Von den 35 Patienten zeigten 22 im Verlauf einen Tumorprogress trotz Therapie. Davon wurden zwölf bestrahlt, drei bestrahlt und mit Temozolomid therapiert, einer mit Gammaknife bestrahlt, einer intensiviert mit Temozolomid und fünf ausschließlich symptomatisch behandelt. Nach Strahlentherapie lag die damit erzielte mediane progressionsfreie Zeit bei fünf Monaten und die „Gesamt“-Überlebenszeit bei zehn Monaten. Als positive prognostische Prädiktoren erwiesen sich das Vorhandensein einer Mutation im IDH1Gen (Isocitratdehydrogenase 1) im Tumorgewebe und das Fehlen eines bilateralen Befalls im MRT.

Schlussfolgerungen: Die Autoren folgern, dass trotz eingeschränkter Vergleichbarkeit mit publizierten Fallserien zur Strahlen- und Chemotherapie mit Temozolomid oder Procarbazin, CCNU plus Vincristin (PCV), die Ergebnisse dieser Phase-II-Studie günstig zu beurteilen sind. Damit darf ihres Erachtens die orale Chemotherapie mit PC als eine etablierte Therapiemöglichkeit gelten oder zumindest als ein Standard für einen prospektiven randomisierten Vergleich mit einer (noch nicht absehbaren) experimentellen Therapie bei dieser Entität.

Kommentar: Die vorliegende Arbeit ist verdienstvoll. 1) Sie legt die erste formale prospektive Therapiestudie bei dieser katastrophalen Tumorerkrankung des Gehirns vor und zeigt damit eine Therapiemöglichkeit auf. 2) Sie bestätigt, dass die Gliomatosis cerebri trotz des Einsatzes aller Therapiemodalitäten eine schlechtere Prognose als anaplastische Gliome hat. 3) Ein Vergleich der Wirksamkeit von "PC" mit (dem wahrscheinlich in der klinischen Praxis häufiger eingesetzten) Temozolomid ist nicht möglich. Für PC liegen jetzt jedoch belastbare Daten vor.

Uwe Schlegel, Bochum 